\title{
Electrochemical Synthesis Of Silver And Gold Nanostructures For Surface-Enhanced Raman Spectroscopy
}

\author{
Pierre Brodard, Mikhael Bechelany, Jamil Elias, Laetitia Philippe and \\ Johann Michler
}

\begin{abstract}
EMPA, Swiss Federal Laboratories for Materials Testing and Research, Laboratory for Mechanics of Materials and Nanostructures, Feuerwerkerstrasse 39, CH-3602 Thun, Switzerland
\end{abstract}

Surface-enhanced Raman spectroscopy (SERS) is a powerful technique for identification of molecules due to the unique set of vibrational modes of any molecular species combined with tremendous signal enhancements observed on the surface of noble metals. ${ }^{1}$ However, SERS can be envisaged as an analytical tool only if substrates with reproducible enhancement can be produced. Recently, new substrates with well-defined nanostructures have been tailored in accordance with a detailed comprehension of the SERS mechanism. ${ }^{2}$

The SERS effect is essentially due to the excitation of localized surface plasmons by light. Therefore, synthetic methods allowing a complete control of the morphology and nature of metallic nanostructures are needed. Two types of fabrication techniques have been proposed: direct-write and natural lithographies. The first offers a good control over the morphology, but its serial nature results in a low throughput whereas a poor chemical and crystalline purity is often observed. ${ }^{3}$ On the other hand, natural lithography is massively parallel, allowing the fast and controlled synthesis of large arrays at low cost. ${ }^{4}$

\section{Hexagonal Pattern of Silver Nanodots and Nanorings}

As a first example, we present the synthesis of large ordered areas of silver nanoparticles on a $\mathrm{Si}(111)$ surface fabricated using electroless metal deposition and the nanospheres lithography method. ${ }^{5}$ The possibility to control the size, the interdistance and the crystallinity of these nanoparticles allowed us to systematically investigate their influence on SERS. Nanodots with diameters well below the wavelength of the laser light $(\lambda=514.5 \mathrm{~nm})$ present a SERS efficiency increasing linearly with size. On the contrary, when the nanodots size approaches $\lambda$, this effect is strongly reduced or even reversed, demonstrating the existence of an ideal size for SERS because too large particles allow the excitation of nonradiative multipoles whereas too small particles lose their electrical conductance and cannot enhance the 
field. Very interestingly, the SERS efficiency for nanorings is almost twice as large as in the case of nanodots of similar size, even though the relative coverage is obviously smaller for nanorings than for nanodots of identical diameters. This clearly indicates an additional effect of the ring geometry. We think that the hole in the middle of the nanorings may act as an additional and controllable hot-spot.
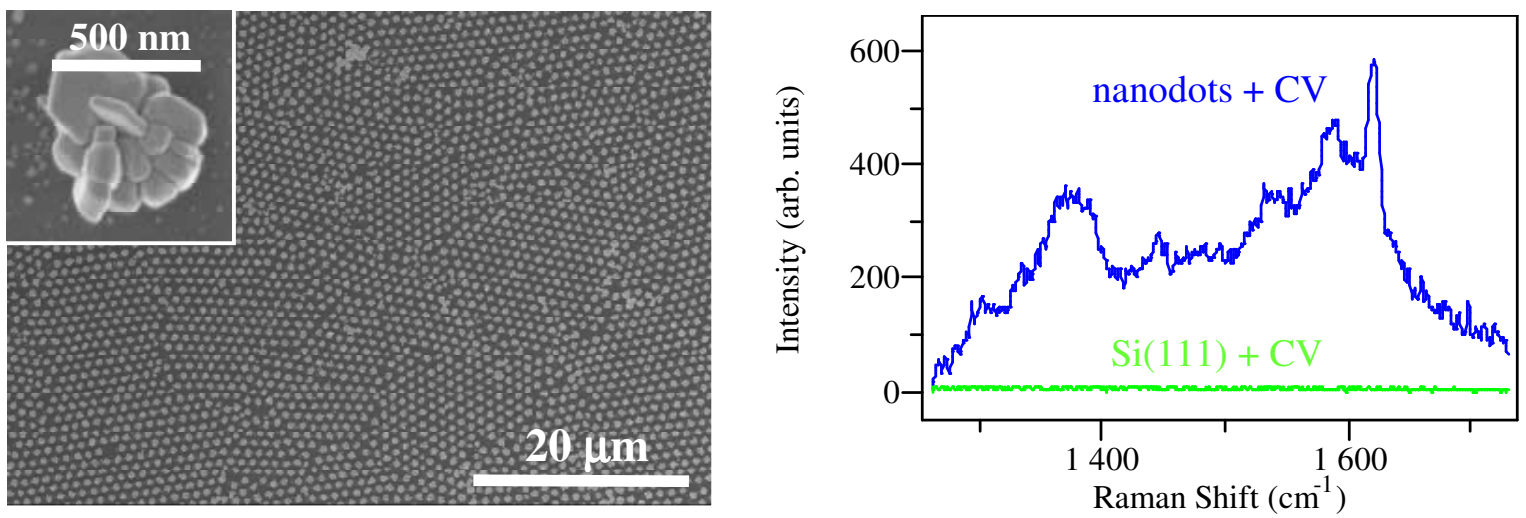

FIGURE 1. SEM image of an isolated pattern of $\mathrm{Ag}$ nanodots formed on $\mathrm{Si}$ and corresponding SERS spectrum of Crystal Violet (CV) as compared to CV on a bare Si(111) substrate.

\section{Gold Flails on Transparent Conductive Oxide Substrates}

As a second recent example, we present a simple electrochemical deposition approach to fabricate, directly on transparent conductive oxide substrates (TCO), dimension-controlled 3D flail-like gold (FG) nano/microstructures with single crystal nanospikes on their surface. The addition of gelatin to the gold bath is shown to play a major role in the synthesis, and we have studied the SERS activity of FGs clusters by probing the Raman response of a deposited monolayer of p-mercaptoaniline (pMA). The SERS effect increases with the number of FGs in the clusters, from no effect on a single-FG cluster to a massive enhancement with $>5$-FGs clusters. This has been observed on FGs of all sizes and shows that the SERS effect is localized in so-called hot-spots, that is contact points between the FGs. In addition, the SERS effect decreases with the FGs size because the size of the spikes is correlated: big FGs have large spikes (size $>\lambda$ ) and therefore cannot come close enough to each other to generate hot-spots, resulting in low SERS activity.

\section{REFERENCES}

1. M. Fleischmann, P. J. Hendra, and A. J. McQuillan, Chem. Phys. Lett. 26, 163 (1974).

2. S. Cintra, M. E. Abdelsalam, P. N. Bartlett, J. J. Baumberg, T. A. Kelf, Y. Sugawara, and A. E. Russell, Faraday Discuss. 132, 191 (2006).

3. G. Laurent, N. Félidj, J. Grand, J. Aubard, G. Lévi, A. Hohenau, F. R. Aussenegg, and J. R. Krenn, Phys. Rev. B 73, 245417 (2006).

4. A. Wei, B. Kim, B. Sadtler, and S. L. Tripp, Chem. Phys. Chem. 2, 743 (2001).

5. M. Bechelany, P. Brodard, L. Philippe, and J. Michler, Nanotechnology 20, 455302 (2009). 